\title{
All-Photonic Molecular Half-Adder
}

Joakim Andréasson, ${ }^{* \dagger}{ }^{\dagger}$ Stephen D. Straight ${ }^{\ddagger}$ Gerdenis Kodis ${ }^{\ddagger}$ Choong-Do Park ${ }^{\ddagger}$ Michael Hambourger, ${ }^{\ddagger}$ Miguel Gervaldo, ${ }^{\ddagger}$ Bo Albinsson, ${ }^{*}{ }^{\dagger}$ Thomas A. Moore, ${ }^{*}{ }^{\star}$ Ana L. Moore ${ }^{*}$, and Devens $^{*}$ Gust**:

Department of Chemical and Biological Engineering, Physical Chemistry, Chalmers University of Technology, SE-412 96 Göteborg, Sweden, and Center for the Study of Early Events in Photosynthesis, Department of Chemistry and Biochemistry, Arizona State University, Tempe, AZ, 85287, USA

E-mail: a-son@chalmers.se or gust@asu.edu

RECEIVED DATE (to be automatically inserted after your manuscript is accepted if required according to the journal that you are submitting your paper to)

Abstract: One molecule acts as both an AND and an XOR Boolean logic gate that share the same two photonic inputs. The molecule comprises a half-adder, adding two binary digits with only light as inputs and outputs, and consists of three covalently linked photochromic moieties; a spiropyran and two quinoline-derived dihydroindolizines. The AND function is based on the absorption properties of the molecule, whereas the XOR function is based on an off-on-off response of the fluorescence to the inputs that results from interchromophore excited state quenching interactions. The half-adder is simple to operate, and can be cycled many times.

\footnotetext{
† Chalmers University of Technology

\# Arizona State University
} 


\section{Introduction}

Many molecules can act as switches, changing structure among two or more states as a result of stimuli. This phenomenon may be harnessed to design molecules that respond to combinations of inputs in ways that correspond to Boolean logic operations, providing a potential basis for molecule-based computing. Arithmetic operations require combinations of two or more logic gates that carry out addition or subtraction. A number of molecular half-adders ${ }^{1-7}$ and half-subtractors ${ }^{4,5,8,9}$ have been described, or discussed theoretically. ${ }^{10,11}$ These function through chemical inputs (e.g., acid, base, ions) that cause the switching operations. Chemical inputs are versatile and simple to implement in demonstrations of principle, but require physical access to the molecules, operate at speeds limited by molecular diffusion, require a solution phase, and lead to an increase in solution volume and buildup of salts or other byproducts after repeated cycling of the device. Molecular switches that use solely light as inputs and outputs avoid these problems. We have reported a variety of all-photonic switches and logic gates based on photochromic molecules. Photochromes photoisomerize between two forms upon exposure to light of various wavelengths. ${ }^{1,12-18}$ We recently reported a photonic half-adder based on two molecular logic gates and a third-harmonic-generating crystal. ${ }^{1}$ Tian and coworkers have described a possible approach to a photochemically-driven half-adder based on a rotaxane, ${ }^{6}$ although the function of the XOR gate portion requires reading different outputs, depending upon which inputs were applied. Here, we report a molecular triad consisting of two different photochromes: a spiropyran and a quinoline-derived dihydroindolizine. Using two inputs of ultraviolet light, the molecule functions as an AND gate via its absorption spectrum and an XOR gate through its fluorescence properties. The XOR gate operates through a non-linear, sometimes called "neuronal," response to light that involves interchromophore excited state quenching. The combination of these two gate functions produces a halfadder with simple optical inputs and readouts.

\section{Results and Discussion}


We will first describe the performance of the half-adder, in order to give the reader a general understanding of how it works, and then discuss the functional details. A Boolean half-adder, characterized by truth Table 1, carries out binary addition of two digits. It consists of an AND logic gate and an XOR gate that share two inputs, $A$ and $B$. These inputs represent two binary numbers $(O$ or 1$)$. The AND gate, which generates the carry digit, yields an on response only when both inputs are on. The XOR (exclusive OR) gate produces the sum digit, and generates an on response when either $A$ or $B$ is on, but not when both inputs are on.

Half-adder molecule 122 (Figure 1) consists of three photochromes linked to a central benzene, which

Table 1. Truth Table for the Half-Adder

\begin{tabular}{ccccc}
\hline Input $A$ & Input $B$ & Output $X$ & Output $Y$ & Binary sum \\
$\lambda=355 \mathrm{~nm}$ & $\lambda=355 \mathrm{~nm}$ & $(A @ 581 \mathrm{~nm})$ & $($ Em. @ $690 \mathrm{~nm})$ & \\
& & AND gate & XOR gate & \\
& & (carry digit) & (sum digit) & \\
\hline 0 & 0 & 0 & & 00 \\
1 & 0 & 0 & 0 & 01 \\
0 & 1 & 0 & 1 & 01 \\
1 & 1 & 1 & 0 & 10
\end{tabular}

functions as an organizational core. One chromophore is a spiropyran (1), and the other two are identical quinoline-derived dihydroindolizines (2). The spiropyran in its closed, thermally stable form (1c) may be photoisomerized with ultraviolet light (e.g. $355 \mathrm{~nm}$ ) to an open merocyanine form (1o), which thermally closes back to 1c. Visible light also causes isomerization to 1c. Each quinoline-derived dihydroindolizine also exists in a thermally stable closed form 2c. Photoisomerization with ultraviolet light (e.g. $355 \mathrm{~nm}$ ) produces an open, zwitterionic form that closes thermally, or with visible irradiation. Ignoring stereoisomerism arising from stereocenters in the spiro forms, $\mathbf{1 2 2}$ can in principle exist in six 
isomeric forms: 1c2c2c, 1c2o2c (where either one of the dihydroindolizines is open), 1c2o2o, 1o2c2c, 10202c, and 102o2o. Only one form, 1c2c2c, is thermally stable, and any of the other isomers will revert to this form in a few minutes at ambient temperatures. Irradiation of $\mathbf{1 2 2}$ at $355 \mathrm{~nm}$ populates all the isomeric forms (Figure 2). Although the details of the kinetic processes are complex, the overall concept is simple: Irradiation at $355 \mathrm{~nm}$ leads to net isomerization in the direction of the arrows in Figure 2, whereas thermal reversion results in net isomerization in the opposite direction. In the dark, the entire population is in state 1c2c2c, at the bottom of the hexagon. Under UV irradiation, the center of the population distribution rises towards the top of the hexagon; at steady-state, the center of this distribution is determined by the equilibria between the thermal and photochemical reactions, which are controlled by the light flux and temperature.

The three chromophores of $\mathbf{1 2 2}$ interact photophysically, and this forms the basis of the half-adder function. The open, merocyanine form of the spiropyran absorbs strongly in the visible $\left(\lambda_{\max }=579 \mathrm{~nm}\right)$, and is the only chromophore of $\mathbf{1 2 2}$ to fluoresce significantly $\left(\lambda_{\max }=640 \mathrm{~nm}\right)$. The open form of the quinoline-derived dihydroindolizine absorbs in the same wavelength region as merocyanine emission $\left(\lambda_{\max }=659 \mathrm{~nm}\right)$, and strongly quenches the merocyanine fluorescence. Thus, of all of the isomeric forms of 122, only 1c2c2c is thermally stable, only $102 c 2 c$ is strongly fluorescent, and only 1o2c2c, 10202c, and 10202o feature the characteristic absorbance of the merocyanine at $579 \mathrm{~nm}$. When 122 is used as a half-adder, inputs $A$ and $B$ are provided by two light beams at $355 \mathrm{~nm}$ with equal intensities. The output of the AND gate $(X)$ is the total absorbance at $581 \mathrm{~nm}$ (mostly due to 1o2c2c, 1o2o2c, and 102020), and the output of the XOR gate $(Y)$ is the fluorescence of 1o2c2c at $690 \mathrm{~nm}$, with excitation at $581 \mathrm{~nm}$. Because the AND and XOR gates have the same inputs and operate concurrently, 122 can function as a half-adder.

The half-adder (Figure 3a) consists of a solution of 122 in a suitable solvent, two 355-nm light sources, and apparatus to measure absorbance at $581 \mathrm{~nm}$, and fluorescence at $690 \mathrm{~nm}$ with $581-\mathrm{nm}$ excitation. (For convenience, only a single light source was used to provide the two inputs in our 
experiments, as described in the Experimental Section.) In AND gate operation, when neither input is on, the molecule is in state 1c2c2c, and no outputs are on. When only one input is turned on, the total merocyanine concentration rises to a steady-state value determined by the light flux and the thermal reversion rates (Figure 2). This gives rise to some absorption at $581 \mathrm{~nm}$, but the total absorbance is below a threshold level, and does not trigger an on response (Figure $3 b$ ). When the second input, $B$, is turned on instead, the same steady-state absorbance is measured, and the gate remains off. However, when both inputs are turned on concurrently, the rate of formation of merocyanine increases, but the rate constants for thermal reversion remain unchanged. The center of the isomer distribution rises farther towards the top of the hexagon in Figure 2, and a new, higher steady-state concentration of merocyanine is reached. The absorbance at $581 \mathrm{~nm}$ increases above the threshold, and the gate produces an on response (Figure 3b). These responses follow the truth table of an AND gate.

The XOR output is merocyanine fluorescence at $690 \mathrm{~nm}$ in 1o2c2c. When 355-nm light is applied to the sample, some 1o2c2c is formed, leading to an increase in fluorescence at $690 \mathrm{~nm}$. However, if the light flux is increased, 10202c, and finally 102020 are generated from 1o2c2c, and these are essentially nonfluorescent due to quenching by the open dihydroindolizine. The result is that as the 355-nm light intensity is increased from zero, the steady-state fluorescence at $690 \mathrm{~nm}$ initially increases, goes through a maximum, and then decreases as the population of quenched isomers increases. In XOR gate operation with two 355-nm inputs, the steady-state emission is therefore significantly greater at the lower light levels generated by one input than at the higher light levels that are produced when both inputs are turned on (Figure 3c). The switching threshold for the XOR gate is exceeded when either input $A$ or input $B$ is applied, but not when both inputs are applied concurrently; this behavior meets the requirements for an XOR gate. After any combination of inputs, the gate can be reset to 1c2c2c thermally, or by irradiating with red light.

From the description above, it is evident that a solution of $\mathbf{1 2 2}$ acts as a half-adder: two binary digits, represented by the two inputs, are added (Table 1). The paragraphs below discuss in detail the 
preparation of the molecule, the photochemical properties of $\mathbf{1 2 2}$ and two model systems, and the performance and resistance to photochemical degradation of the half-adder.

Synthesis. The core of $\mathbf{1 2 2}$ was prepared from 5-aminoisophthalic acid, whose amino functionality was protected with a $t$-butoxycarbonyl group. The spiropyran acid was prepared as previously described. ${ }^{19}$ The precursor of the quinoline-derived dihydroindolizine moieties of 122, 6-hydroxy-spiro[9H-fluorene-9,3' (3'aH)-pyrrolo[1,2-a]-quinoline]-1',2'-dicarbonitrile, was prepared using procedures adapted from reported preparations of related compounds. ${ }^{20}$ The quinoline-derived dihydroindolizines were coupled to amine-protected 5-aminoisophthalic acid using 3-chloro-4,6-dimethoxy-1,3,5-triazine and N-methylmorpholine. After removal of the protecting group from the resulting dyad, the spiropyran acid was attached using 3-chloro-4,6-dimethoxy-1,3,5-triazine and N-methylmorpholine. Model compounds 3 and 4 (Figure 4) were prepared using similar procedures. Additional information concerning the synthesis and spectroscopic techniques for all compounds is given in the Supporting Information.

Photochemistry of model compounds. In order to understand the photochemical behavior of the triad, we investigated model spiropyran 3 and model dihydroindolizine $\mathbf{4}$ spectroscopically. The steadystate absorption and emission studies reported below were performed on solutions $\left(\sim 1 \times 10^{-5} \mathrm{M}\right)$ in 2 methyltetrahydrofuran at $22{ }^{\circ} \mathrm{C}$, with optical path lengths of $1.0 \mathrm{~cm}$ and sample volumes of $1.5 \mathrm{~mL}$.

Spiropyran 3. As is the case with other spiropyrans, $\mathbf{3}$ is thermally stable in the closed, spiro form $\mathbf{3 c}$ (Figure 4). The absorption spectrum of 3c (Figure 5a) features maxima at 335 and $271 \mathrm{~nm}$. There is no absorption in the visible region of the spectrum. Irradiation at $355 \mathrm{~nm}$ causes isomerization to a photostationary distribution containing mainly the merocyanine form 3o (see Figure 1). The merocyanine has absorption maxima at 593, 334, and $274 \mathrm{~nm}$.

Spiro form 3c does not fluoresce significantly. Merocyanine 3o does fluoresce, with $\lambda_{\max }=664 \mathrm{~nm}$ $\left(\Phi_{\mathrm{f}}=\sim 0.03\right.$ ) (Figure 5a). Pump-probe transient absorption investigations were performed on 3o. A 2methyltetrahydrofuran solution of $\mathbf{3}$ was constantly irradiated at $366 \mathrm{~nm}$ to maintain a photostationary 
distribution containing mainly the open form, and the solution was excited at $585 \mathrm{~nm}$ with $\sim 100$ fs laser pulses. The transient absorption was probed at $720 \mathrm{~nm}$ (Figure 6). Three components were observed: a major component (67\%) with a lifetime of $45 \pm 1$ ps, a $140 \pm 10$ ps component (19\%), and a $0.42 \pm 0.05$ ps component with an amplitude of $-14 \%$ (grow-in of stimulated emission amplitude with time). Similar results showing the presence of and interconversions between isomers of merocyanines have been observed for other spiropyrans. ${ }^{18,21-28}$ Based on studies of a similar molecule, ${ }^{18}$ we can assign the 45 ps lifetime in 30 to the major isomer, which absorbs at $593 \mathrm{~nm}$ and emits relatively strongly at $664 \mathrm{~nm}$.

Quinoline-derived dihydroindolizine 4. As shown in Figure 5a, the thermally stable, closed form of the dihydroindolizine, 4c, has absorption maxima in the UV at 393 and $338 \mathrm{~nm}$. After irradiating at 355 $\mathrm{nm}$ to generate a photostationary distribution rich in the open, zwitterionic isomer $\mathbf{4 o}$, new bands appear at 666 and $484 \mathrm{~nm}$. The 40 isomer does not display any detectable fluorescence. Transient absorption experiments in 2-methyltetrahydrofuran yielded the transient at $720 \mathrm{~nm}$ shown in Figure 6 . This is ascribed to a mixture of stimulated emission, ground state bleaching, and induced absorption from an unrelaxed ground state. The first excited singlet state of $\mathbf{4 o}$ decays with a time constant of $0.84 \pm 0.02$ ps. A minor component of $2.6 \pm 0.02 \mathrm{ps}$ is ascribed to induced absorption from an unrelaxed ground state.

Photochemistry of the triad. The absorption spectrum of 1c2c2c in 2-methyltetrahydrofuran (Figure 5b) shows maxima at 390, 363 (sh), and $272 \mathrm{~nm}$ (maximum not shown in Figure). The features at 363 and $272 \mathrm{~nm}$ are similar to the absorption bands of model spiropyran 3c, whereas the $390 \mathrm{~nm}$ band is clearly related to the $393 \mathrm{~nm}$ band in dihydroindolizine model $\mathbf{4 c}$ (Figure 5a). Thus, the triad absorption is consistent with a superposition of the bands of the component chromophores. Irradiation of the sample of 1c2c2c at $355 \mathrm{~nm}$ results in the appearance of new bands at 659, 579 and $485 \mathrm{~nm}$ (Figure 5b). The 659 and $485 \mathrm{~nm}$ bands are similar to those of the open form of the dihydroindolizine model 40 whereas the $579 \mathrm{~nm}$ band is similar to the 593-nm band of 3o. Thus, irradiation of 1c2c2c with ultraviolet light generates a photostationary distribution containing significant amounts of the open forms of the chromophores. The amplitude in the $700-750 \mathrm{~nm}$ region is a function of the amount of open dihydroindolizine present, whereas the amplitude in the 580-nm region reflects mainly the amount 
of the merocyanine form of the spiropyran. The only fluorescence emission observed from 122 is from the merocyanine form of the spiropyran, with a maximum at $640 \mathrm{~nm}$.

Isomerization kinetics. The thermal closing kinetics of the photochromes in $\mathbf{1 2 2}$ were investigated. A sample of 1c2c2c in 2-methyltetrahydrofuran at $22{ }^{\circ} \mathrm{C}$ was irradiated at $355-\mathrm{nm}$ to prepare a solution enriched in the open isomers, the light was extinguished, and the absorbance as a function of time was monitored. Time constants for the exponential thermal isomerizations were $160 \mathrm{~s}$ for the spiropyran and $189 \mathrm{~s}$ for the dihydroindolizine. Both isomerization rates significantly increased with visible light irradiation $\left(580 \leq \lambda \leq 900 \mathrm{~nm}, \sim 50 \mathrm{~mW} / \mathrm{cm}^{2}\right)$ because photochemical closing contributed. Photochemical opening rates were studied using $355 \mathrm{~nm}$ light from a Nd:YAG pulsed laser operated at $10 \mathrm{~Hz}$. The average total power of the beam striking the sample was $28 \mathrm{~mW}$. Time constants for opening of the spiropyran and dihydroindolizine were 4.4 and $5.1 \mathrm{~s}$, respectively. When the average light flux was reduced by a factor of two by slowing the laser repetition rate to $5 \mathrm{~Hz}(14 \mathrm{~mW}$ average total power), the corresponding time constants were $7.9 \mathrm{~s}$ and $8.5 \mathrm{~s}$. At the lower light flux, the photoisomerization rates are not decreased by a factor of two because thermal closing competes with the photochemical opening, and the thermal rate constants do not change with light flux.

Absorbance as a function of isomerization rates. Figure 7 a shows the time dependence of the absorbance of $\mathbf{1 2 2}$ in 2-methyltetrahydrofuran under exposure to 355-nm light. At zero time, the sample consisted completely of 1c2c2c. The solid line shows the absorbance at $581 \mathrm{~nm}$, where most of the absorbance is due to the merocyanine form of the spiropyran, when the sample was exposed to 355-nm laser pulses at $5 \mathrm{~Hz}(14 \mathrm{~mW}$ average power). The absorbance rises from virtually zero when the light is turned on at $0 \mathrm{~s}$, reaching a steady-state value after about $35 \mathrm{~s}$. The absorbance at any time reflects the sum of the concentrations of the three isomers of $\mathbf{1 2 2}$ that contain the merocyanine. When the light is first turned on, the population distribution (Figure 2) favors 1o2c2c and 1c2o2c. As irradiation is continued, the population shifts towards the top of the hexagon in Figure 2, and 10202c and 102020 make greater contributions to the absorbance at $581 \mathrm{~nm}$. After $35 \mathrm{~s}$, a steady-state distribution is 
attained. The fraction of each of the six isomers is determined not only by the various rate constants for photoinduced opening and closing, but also the rate constants for thermal closing.

The dotted line in Figure 7a shows the results of a similar experiment carried out with the laser at a repetition rate of $10 \mathrm{~Hz}(28 \mathrm{~mW}$ average power). The concentration of merocyanine initially rises at about twice the rate observed in the first experiment, as expected. The steady-state concentration is higher because the rates of photochemical opening of the spiropyran (and photochemical closing) increase due to the doubled laser power, but the thermal closing rate constants are not changed, moving the steady-state distribution of isomers farther toward the top of the hexagon. As will be discussed later, this phenomenon forms the basis of the AND gate function.

Fluorescence as a function of isomerization rates. Figure $7 \mathrm{~b}$ shows the results of a similar experiment in which the emission intensity of the merocyanine at $690 \mathrm{~nm}$ (excitation at $581 \mathrm{~nm}$ ) was monitored. Prior to excitation, there is no appreciable fluorescence because essentially no merocyanine is present. With 355-nm excitation at $5 \mathrm{~Hz}$ (solid line), the emission intensity increases rapidly as some of the sample is converted to $\mathbf{1 0 2 c 2 c}$, which is the only isomer that is significantly fluorescent. After about $3 \mathrm{~s}$ the emission intensity begins to decrease because $102 \mathbf{c} 2 \mathbf{c}$ is being converted into $102 \mathbf{2} 2 \mathrm{c}$, some of which in turn is converted to 102020. Both of these latter isomers are virtually non-fluorescent due to quenching of the merocyanine excited states by the open dihydroindolizine moieties. After about $30 \mathrm{~s}$, a constant emission level is reached, which is established by the steady-state distribution of the population among the six isomers. Thus, upon exposure to $355-\mathrm{nm}$ light, emission from 122 increases to a maximum and then decreases to a steady-state value. This kind of "off-on-off" behavior has been termed "neuronal," because it resembles that of the chemical synapses of neurons. Several examples of neuronal behavior in molecular systems have been reported. ${ }^{29-34}$

When the average light flux is doubled by doubling the repetition rate of the laser to $10 \mathrm{~Hz}$, the fluorescence intensity as a function of time is different (dotted line, Figure 7b). Due to the higher light flux, the emission at $690 \mathrm{~nm}$ increases more rapidly than in the 5-Hz experiment because 1o2c2c is formed more rapidly from 1c2c2c. However, the intensity maximum is reached sooner, as the rates of 
formation of 10202c and 102020 are also increased. Most importantly, the steady-state emission intensity is lower than that observed with lower light flux. The reason is that with the higher flux, the steady-state distribution of isomers contains a higher fraction of the quenched isomers 1o202c and 102020, which are formed at the expense of the emissive 1o2c2c. This inverse behavior, where increased light intensity leads to lower emission intensity, forms the basis of the XOR gate.

The quenching process. It is clear from the data that the open forms of the dihydroindolizine of 122 quench the fluorescence of the merocyanine form of the spiropyran. Information concerning the kinetics of this quenching was obtained from time-resolved transient absorbance studies with excitation at $585 \mathrm{~nm}$ by ca. 100 fs laser pulses, during which time the sample was continuously irradiated at 366 $\mathrm{nm}$ in order to assure a population of isomers containing the merocyanine and the open forms of the dihydroindolizines. The population of the merocyanine first excited singlet state was monitored via its stimulated emission at $720 \mathrm{~nm}$ (Figure 6). ${ }^{18}$ Because the steady-state population contains six different forms of the triad, and because, as discussed above, the model spiropyran and dihydroindolizine molecules themselves display multiple excited state decay processes, it is not practical to interpret these data in terms of specific concentrations and rate constants. However, it is obvious from Figure 6 that linking the chromophores to form the triad does introduce new decay components that are not present in the model compounds. In particular, a new component with a lifetime on the 3 ps time scale is observed. The data for the first $10 \mathrm{ps}$ of the transient decay could be fitted with three exponential processes: 45 ps (40\% of the decay, fixed lifetime based on results for model merocyanine 3o), 0.84 ps (30\%, fixed lifetime based on model open dihydroindolizine 4o) and $3 \pm 1$ ps (30\%). The 3 -ps component is ascribed to quenching of the merocyanine excited singlet state. Because the relative populations of 1o2o2c and 102020 under the conditions of the experiment are not known, we do not know whether this value is characteristic of one of these isomers, or an unresolved mixture of time constants for both. In addition, the above analysis is oversimplified, as the decay kinetics, especially at longer times (not shown), contain additional components, as discussed above. The important point is that the results are consistent with the strong quenching of the merocyanine excited singlet state that was discussed above, because 
the relevant lifetimes of the merocyanine excited states observed in model compound 3o were 45 and 140 ps.

The nature of the quenching process is not immediately apparent. Singlet-singlet energy transfer or photoinduced electron transfer are the most likely mechanisms, and the spectroscopic data do not allow us to strictly rule out either process. However, photoinduced electron transfer seems unlikely. The first oxidation potential of merocyanine 30 is $1.20 \mathrm{~V}$ vs SCE, and the first reduction potential is $-1.25 \mathrm{~V}$. $^{18}$ Cyclic voltammetric measurements of model dihydroindolizine 5o yielded a first oxidation potential of $0.75 \mathrm{~V}$, and a first reduction potential of $-0.70 \mathrm{~V}$. Thus, the lowest-energy charge-separated state in 122 would contain a merocyanine radical cation and an open dihydroindolizine radical anion, and would have an energy of ca. $1.9 \mathrm{eV}$ above that of the ground state. The energy of the excited state of the merocyanine is ca. $2.0 \mathrm{eV}$. Photoinduced electron transfer would have a thermodynamic driving force of ca. $100 \mathrm{mV}$. It seems unlikely that this process would occur on the 3 ps time scale. On the other hand, the excellent overlap between the emission of the merocyanine and the absorption of the open dihydroindolizine (Figure 5), coupled with the short distance between the chromophores, suggests that singlet-singlet energy transfer by the Förster mechanism would be extremely efficient. We therefore expect that energy transfer is the most likely quenching process. In any case, the nature of the quenching is not actually relevant for the performance of the device, which simply requires that quenching of some sort occur.

Performance as a half-adder. Given the discussion of triad photochemistry presented above, it is clear that $\mathbf{1 2 2}$ can act as a single-molecule half-adder with all-photonic inputs and outputs. As illustrated in Figure 3a, the two inputs, shared by both the AND and XOR gate functions, are light at $355 \mathrm{~nm}$. In principle, these could be two steady-state light sources of equal intensity, but in this work we used a pulsed laser operating at $5 \mathrm{~Hz}$ (for input $A$ or input $B ; 14 \mathrm{~mW}$ average power) or $10 \mathrm{~Hz}$ (for both inputs on concurrently, $28 \mathrm{~mW}$ average power) for the sake of convenience. The output for the AND gate is the absorbance of the merocyanine in $102 \mathbf{c} 2 \mathrm{c}, 102 \mathrm{o} 2 \mathrm{c}$, and $10202 \mathrm{o}$ monitored at $581 \mathrm{~nm}$. The output for the XOR gate is emission of the merocyanine in 1o2c2c, excited at $581 \mathrm{~nm}$ and monitored at $690 \mathrm{~nm}$. 
Although in principle both outputs could be induced by the same 581-nm analyzing light and measured with photodiodes having suitable geometries and interference filters, we measured them separately using a fluorimeter and absorbance spectrophotometer. In our implementation of the half-adder, both outputs were obtained $6.5 \mathrm{~s}$ after the input(s) were turned on, as this arrangement was convenient and gave good signal-to-noise ratios. From Figure 7 it is clear that the outputs could have been read at any later time, as long as the inputs were left on until the outputs were determined.

In operation, the initial state of the half-adder is 1c2c2c. This is the only significant species present in the dark at thermal equilibrium, and the system may be reset to this state after application of any combination of inputs by irradiation with red light $(580 \leq \lambda \leq 900 \mathrm{~nm})$. In this state, no visibleabsorbing isomers are present, and both outputs are off, representing the addition of 0 and 0 to give 00 in binary (Figures $3 \mathrm{~b}$ and c). If either input is turned on, the absorbance at $581 \mathrm{~nm}$ rises to a steady-state value, signaling formation of merocyanine. However, the threshold for an on response of the AND gate is set at a higher amplitude than this, so the AND gate remains off. With one input on, the emission of the merocyanine rises to a value above the threshold, signaling an on response of the XOR gate. Thus, the half-adder adds 0 and 1 (or 1 and 0 ) to give 01 . Finally, if both inputs are turned on, the absorbance at 581 increases to a value above the threshold because additional merocyanine-containing species are produced. The AND gate yields an on output. The fluorescence at 690, however, is lower than that observed for only one input, due to fluorescence quenching, and the XOR output is off. Thus, the halfadder output is 10 , or 2 in base-10 arithmetic. Figures $3 \mathrm{~b}$ and c show the actual response levels attained in these experiments. The absorbance and emission data are essentially noise free under these conditions (Figure 7).

Cycling and photodecomposition. To be useful in any real-world application, a molecular arithmetic device must be capable of cycling through its various states numerous times. Although the intent of this work is to demonstrate function, and not to produce a technological device, we did investigate the stability of 122 when cycling. Figure 8a shows the absorbance at $581 \mathrm{~nm}$ during a sequence of 10 reset $\left(580 \leq \lambda \leq 900 \mathrm{~nm}\right.$, ca. $\left.50 \mathrm{~mW} / \mathrm{cm}^{2}, 10 \mathrm{~min}\right)$, single input $(355 \mathrm{~nm}$ laser pulses at $5 \mathrm{~Hz}, 14 \mathrm{~mW}$ average 
power, $6.5 \mathrm{~s})$, and dual input $(355 \mathrm{~nm}$ laser pulses at $10 \mathrm{~Hz}, 28 \mathrm{~mW}$ average power, $6.5 \mathrm{~s})$ exposures and readout operations. There is little or no degradation within the variations of the measurements. Figure $8 \mathrm{~b}$ shows the emission at $690 \mathrm{~nm}$ following the same series of inputs and resetting operations. Again, little or no degradation is observed. Thus, the material is stable under these conditions when rigorously deoxygenated.

\section{Conclusions}

Triad 122 functions as a one-molecule Boolean half-adder with all-photonic inputs and outputs. It is stable and capable of cycling many times. The instrumentation required for operation is very simple, consisting, in principle, of two identical steady-state light sources as inputs, a single light source for readout, and two diode detectors to measure absorbance and emission changes at suitable wavelengths. Incorporation of both the AND and XOR functions into the same molecule greatly simplifies the system. The chemical linkage between the chromophores permits rapid and efficient quenching of the merocyanine excited state by the open dihydroindolizine, making the XOR function possible.

The cycle times are dependent upon the inputs. The time constants for the various photochemical reactions are on the ns time scale or less, and the time required to reach steady state depends upon the intensity of the exciting light. In the experiments reported here, total irradiation times (with 5 ns pulses) were on the order of a few hundred ns. If readout is performed only after steady-state conditions are reached (Figure 7), it is clear that thermal isomerization times will limit the device speed, and these will be governed by the temperature. However, from Figure 7 it is apparent that there is no need to wait until equilibrium is achieved in order to obtain adequate signal-to-noise ratios. Under the conditions employed, clear response differences are apparent in as few as $6 \mathrm{~s}$ after inputs are turned on. One disadvantage of this system is that the inputs must be on during readout, or turned off immediately prior to readout. Because of thermal reversion to the off form, the molecule does not record its state for more than a few minutes at room temperature after application of inputs ceases. 
The system has several advantages over molecular arithmetic systems that depend upon addition of chemicals for some or all of the inputs. No physical access for materials to the volume element containing the half-adder is required, fluid solutions are not required, diffusional processes do not play a role, and resetting is possible without the buildup of side products and dilution that could eventually interfere with the operation of the system. Although this triad successfully demonstrates the function of a molecular half-adder, the usefulness of such molecular logic in real-world applications has yet to be determined.

Acknowledgment. This work was supported by the Swedish Research Council (VR) and the U. S. National Science Foundation (CHE-0352599). This is publication 669 from the ASU Center for the Study of Early Events in Photosynthesis.

Supporting Information Available: Synthesis and characterization of the compounds described herein and the conditions for performance of the optical studies are available free of charge via the Internet at http://pubs.acs.org. 


\section{References}

(1) Andréasson, J.; Kodis, G.; Terazono, Y.; Liddell, P. A.; Bandyopadhyay, S.; Mitchell, R. H.;

Moore, T. A.; Moore, A. L.; Gust, D. J. Am. Chem. Soc. 2004, 126, 15926-15927.

(2) de Silva, A. P.; McClenaghan, N. D. J. Am. Chem. Soc. 2000, 122, 3965-3966.

(3) Guo, X.; Zhang, D.; Zhang, G.; Zhu, D. J. Phys. Chem. B 2004, 108, 11942-11945.

(4) Margulies, D.; Melman, G.; Felder, C. E.; Arad-Yellin, R.; Shanzer, A. J. Am. Chem. Soc. 2004, $126,15400-15401$.

(5) Margulies, D.; Melman, G.; Shanzer, A. Nature Materials 2005, 4, 768-771.

(6) Qu, D. H.; Wang, Q. C.; Tian, H. Angew.e Chem. Int. Ed. 2005, 44, 5296-5299.

(7) Stojanovic, M. N.; Stefanovic, D. J. Am. Chem. Soc. 2003, 125, 6673-6676.

(8) Coskun, A.; Deniz, E.; Akkaya, E. U. Org. Lett. 2005, 7, 5187-5189.

(9) Langford, S. J.; Yann, T. J. Am. Chem. Soc. 2003, 125, 11198-11199.

(10) Remacle, F.; Speiser, S.; Levine, R. D. J. Phys. Chem. B 2001, 105, 5589-5591.

(11) Remacle, F.; Weinkauf, R.; Levine, R. D. J. Phys. Chem. A 2006, 110, 177-184.

(12) Gust, D.; Moore, T. A.; Moore, A. L. Chem. Commun. 2006, 2006, 1169-1178.

(13) Andréasson, J.; Terazono, Y.; Albinsson, B.; Moore, T. A.; Moore, A. L.; Gust, D. Angew. Chem. Int. Ed. 2005, 44, 7591-7594.

(14) Terazono, Y.; Kodis, G.; Andréasson, J.; Jeong, G.; Brune, A.; Hartmann, T.; Dürr, H.; Moore, A. L.; Moore, T. A.; Gust, D. J. Phys. Chem. B 2004, 108, 1812-1814.

(15) Liddell, P. A.; Kodis, G.; Andréasson, J.; de la Garza, L.; Bandyopadhyay, S.; Mitchell, R. H.; Moore, T. A.; Moore, A. L.; Gust, D. J. Am. Chem. Soc. 2004, 126, 4803-4811.

(16) Straight, S. D.; Andréasson, J.; Kodis, G.; Bandyopadhyay, S.; Mitchell, R. H.; Moore, T. A.; Moore, A. L.; Gust, D. J. Am. Chem. Soc. 2005, 127, 9403-9409.

(17) Straight, S. D.; Andréasson, J.; Kodis, G.; Moore, A. L.; Moore, T. A.; Gust, D. J. Am. Chem. Soc. 2005, 127, 2717-2724. 
(18) Bahr, J. L.; Kodis, G.; de la Garza, L.; Lin, S.; Moore, A. L.; Moore, T. A.; Gust, D. J. Am. Chem. Soc. 2001, 123, 7124-7133.

(19) Garcia, A. A.; Cherian, S.; Park, J.; Gust, D.; Jahnke, F.; Rosario, R. J. Phys. Chem. A 2000, 104, 6103-6107.

(20) Dürr, H. Photochromism of dihydroindolizines and related systems; In Organic Photochromic and Thermochromic Compounds; Crano, J. C., Guglielmetti, R. J., eds. Plenum Press: New York, 1999; pp 223-266.

(21) Wohl, C. J.; Kuciauskas, D. J. Phys. Chem. B 2005, 109, 22186-22191.

(22) Heiligman-Rim, R.; Hirshberg, Y.; Fischer, E. J. Phys. Chem. 1962, 66, 2465-2477.

(23) Takahashi, H.; Yoda, K.; Isaka, H.; Ohzeki, T.; Sakaino, Y. Chem. Phys. Lett. 1987, 140, 90-94.

(24) Hobley, J.; Malatesta, V. Phys. Chem. Chem. Phys. 2000, 2, 57-59.

(25) Abel, Y.; Nakao, R.; Horii, T.; Okada, S.; Irie, M. J. Photochem. Photobiol. A 1996, 95, 209214.

(26) Cottone, G.; Noto, R.; La Manna, G.; Fornili, S. L. Chem. Phys. Lett. 2000, 2000, 51-59.

(27) Wilkinson, F.; Worrall, D. R.; Hobley, J.; Jansen, L.; Williams, S. L.; Langley, A. J.; Matousek, P. J. Chem. Soc. , Faraday Trans. 1996, 92, 1331-1336.

(28) Marevtsev, V. S.; Zaichenko, N. L. J. Photochem. Photobiol. A 1997, 104, 197-202.

(29) Callan, J. F.; de Silva, A. P.; McClenaghan, N. D. Chem. Commun. 2004, 2048-2049.

(30) deSilva, A. P.; Gunaratne, H. Q. N.; McCoy, C. P. Chem. Commun. 1996, 2399-2400.

(31) Fabbrizzi, L.; Gatti, F.; Pallavicini, P.; Parodi, L. New J. Chem. 1998, 22, 1403-1407.

(32) Gunnlaugsson, T.; Leonard, J. P.; Senechal, K.; Harte, A. J. J. Am. Chem. Soc. 2003, 125, 12062-12063.

(33) Pallavicini, P.; Amendola, V.; Massera, C.; Mundum, E.; Taglietti, A. Chem. Commun. 2002, 2452-2453.

(34) Pina, F.; Melo, M. J.; Maestri, M.; Passaniti, P.; Balzani, V. J. Am. Chem. Soc. 2000, 122, 44964498. 


\section{Figure Captions}

Figure 1. Structure of triad 122 and isomerization of the spiropyran (1) and quinoline-derived dihydroindolizine (2) photochromes.

Figure 2. Graph of isomerization pathways for 122. Net photochemical isomerization under the influence of UV light occurs in the direction of the arrows. Thermal isomerization and photoisomerization promoted by visible light occurs in the opposite direction.

Figure 3 (a) Schematic diagram of the half-adder. (b) Actual performance of the AND gate function under the application of various combinations of inputs. (c) Performance of the XOR gate function. For signal-to-noise ratios in the absorption and emission readout processes, and for reproducibility of the data from one experiment to another, see Figures 7 and 8, respectively.

Figure 4. Structures of the closed, spiro forms of model spiropyran 3c and model quinoline-derived dihydroindolizines $\mathbf{4 c}$ and $\mathbf{5 c}$. The structures of the open forms $(\mathbf{3 o}, \mathbf{4 o}, \mathbf{5 0})$ are analogous to those of the corresponding structures in $\mathbf{1 0}$ and $\mathbf{2 0}$ (Figure 1).

Figure 5. (a) Absorption spectra of 3c (red dots), a photostationary distribution containing mainly 3o (red line), 4c (green dots), a photostationary distribution containing mainly 4o (green line), and emission spectrum of 30 (red dashes). (b) Absorption spectra of 1c2c2c (blue line), and the same solution after irradiation at $355 \mathrm{~nm}$ to generate a photostationary distribution rich in the open forms of the triad (red line).

Figure 6. Transient absorption kinetics at $720 \mathrm{~nm}$ following excitation with a $585 \mathrm{~nm}$, ca. $100 \mathrm{fs}$, laser pulse during constant illumination of the sample at $366 \mathrm{~nm}$. Data for model compounds 3 ( $\square$ ) and 4 (०) and triad $122(\diamond)$ are shown. The figure shows the negative of the absorbance change on a natural logarithm scale for the first 7 ps of the observation period. The solid line is a multi-exponential fit to the 
data for 122, as discussed in the text. The $\Delta A$ values have been normalized for ease in comparison of the kinetics; the actual values are $\leq-0.03$.

Figure 7. (a) Absorbance of a solution of 122 in 2-methyltetrahydrofuran at $581 \mathrm{~nm}$ as a function of irradiation time with $355 \mathrm{~nm}$ laser pulses having a repetition rate of $5 \mathrm{~Hz}\left({ }_{-}\right)$or $10 \mathrm{~Hz}(\bullet \bullet \bullet)$. (b) Fluorescence of a solution of 122 in 2-methyltetrahydrofuran at $690 \mathrm{~nm}\left(\lambda_{\mathrm{ex}}=581 \mathrm{~nm}\right)$ as a function of irradiation time with $355 \mathrm{~nm}$ laser pulses having a repetition rate of $5 \mathrm{~Hz}(-)$ or $10 \mathrm{~Hz}(\bullet \bullet \bullet)$.

Figure 8. Cycling of the triad. (a) Absorbance at $581 \mathrm{~nm}$ following reset $(580 \leq \lambda \leq 900 \mathrm{~nm}$, ca. 50 $\left.\mathrm{mW} / \mathrm{cm}^{2}, 10 \mathrm{~min}\right)$ (black), irradiation with one input $(355 \mathrm{~nm}$ laser pulses at $5 \mathrm{~Hz}, 14 \mathrm{~mW}$ average power, $6.5 \mathrm{~s})$ (red) and irradiation with both inputs $(355 \mathrm{~nm}$ laser pulses at $10 \mathrm{~Hz}, 28 \mathrm{~mW}$ average power, $6.5 \mathrm{~s})$ (green). (b) Emission at $690 \mathrm{~nm}$ (581 nm excitation) following the same sequence of irradiations. 
Figure 1
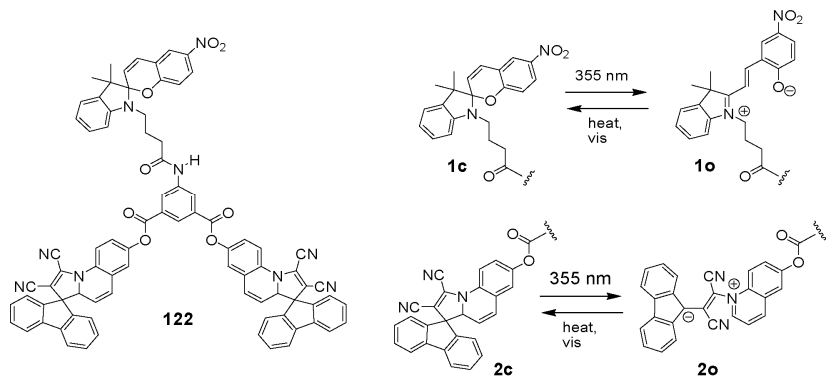
Figure 2

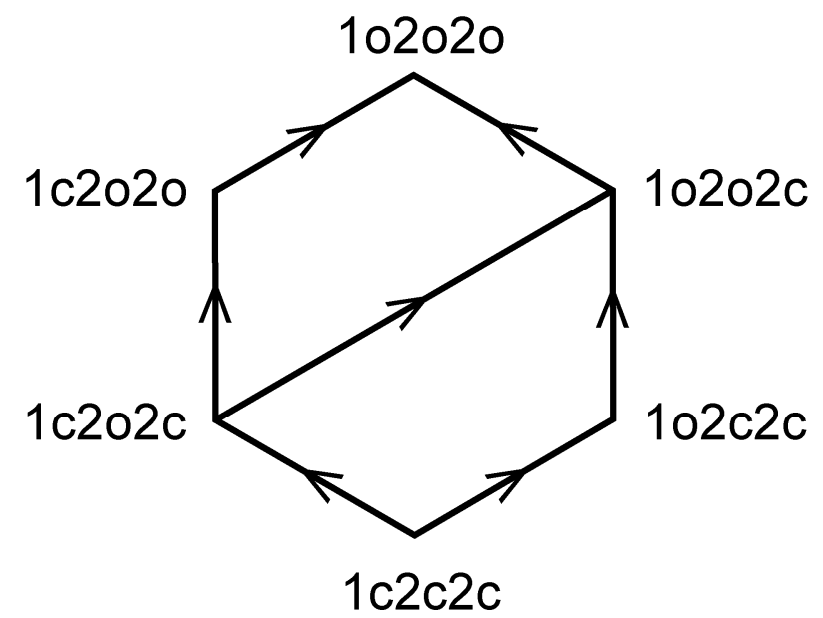


Figure 3
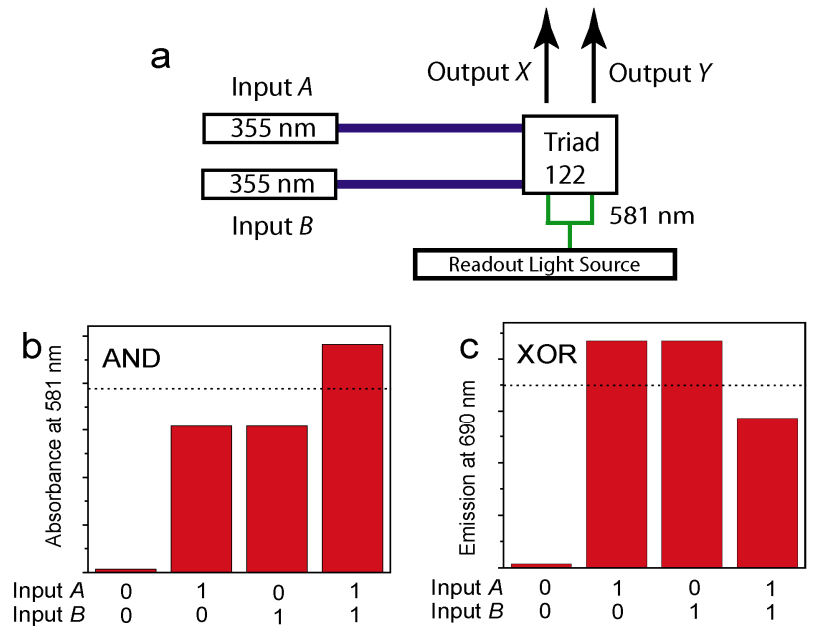
Figure 4

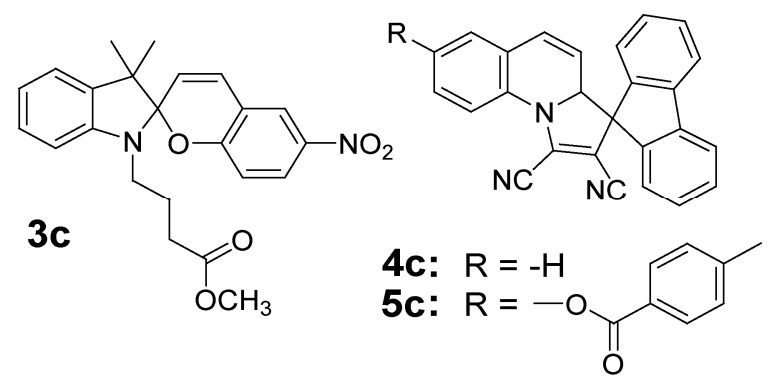


Figure 5
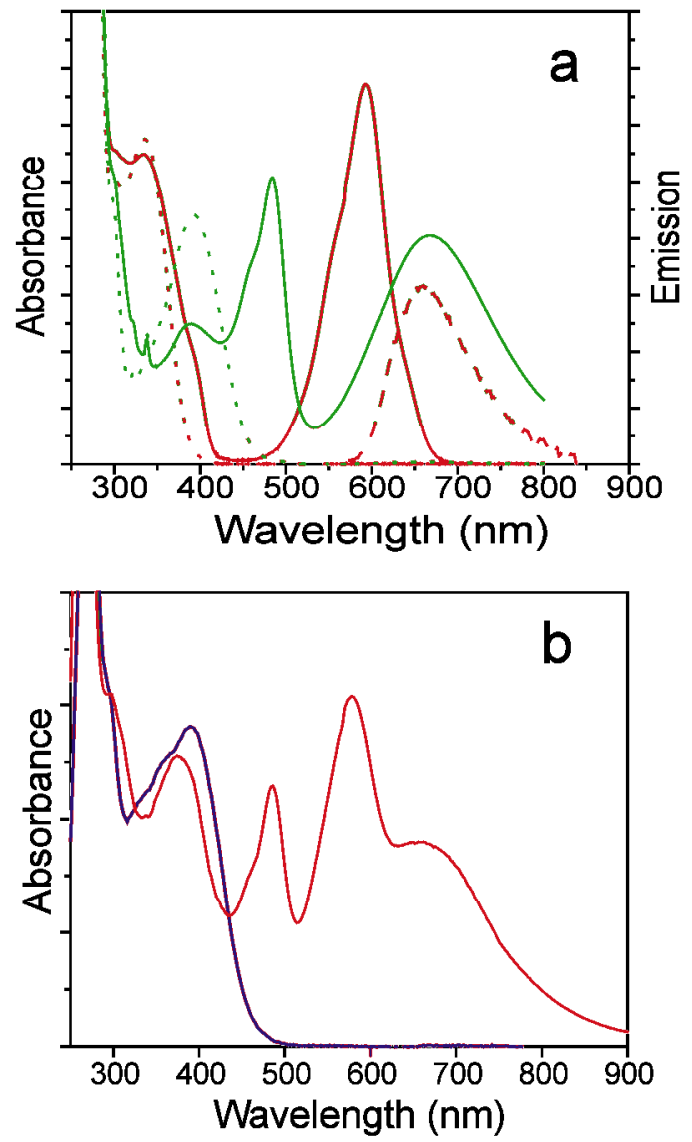
Figure 6

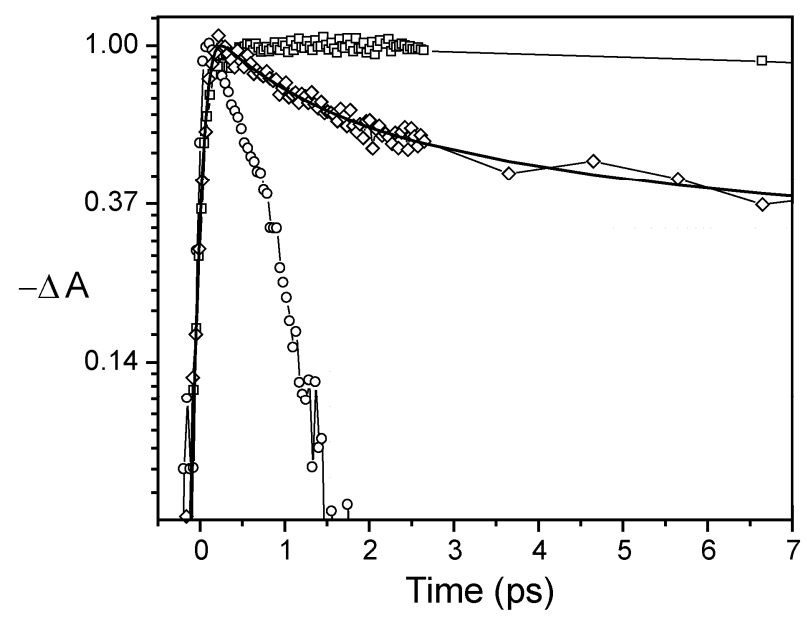


Figure 7
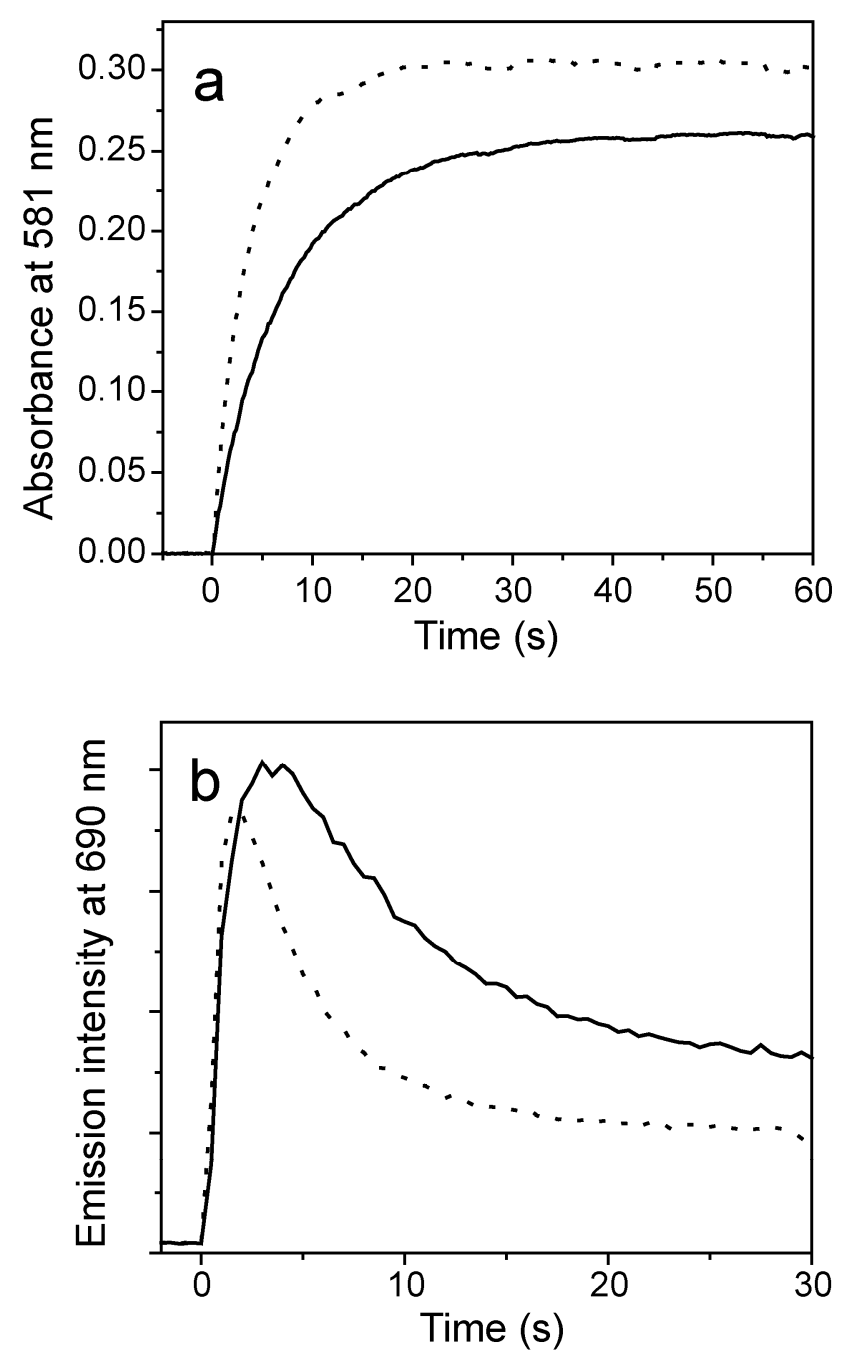
Figure 8
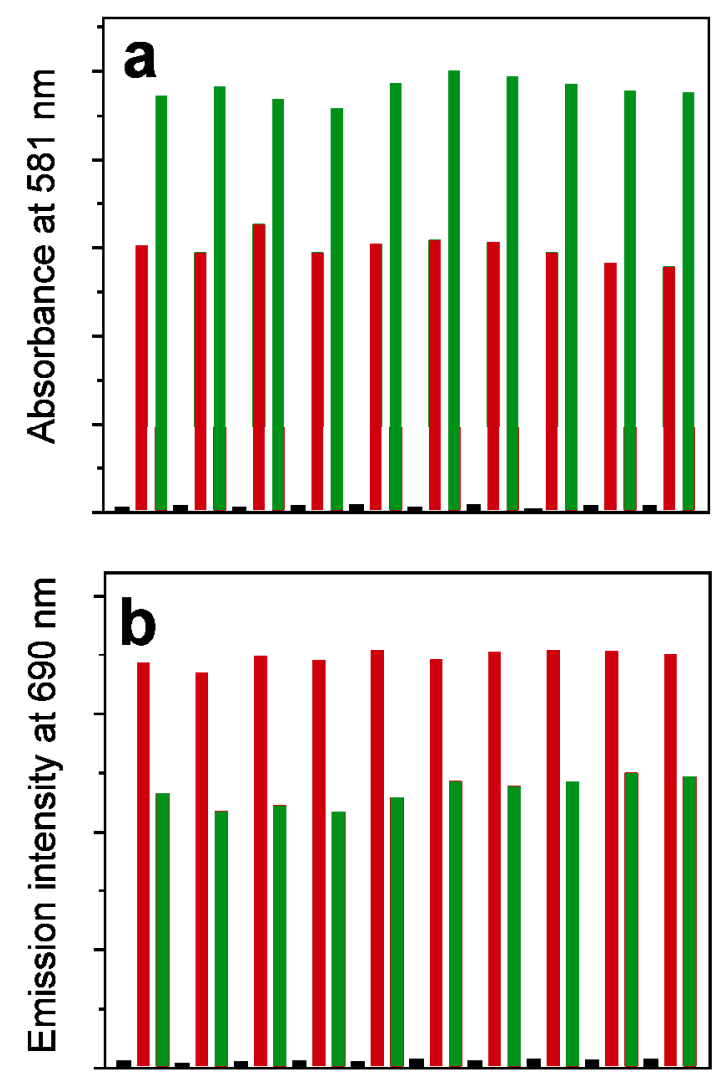
TOC Graphic

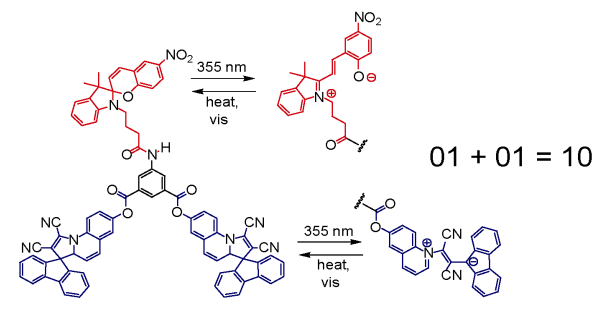

